\title{
CORRIGENDUM
}

\section{Semi-metallic polymers}

Olga Bubnova, Zia Ullah Khan, Hui Wang, Slawomir Braun, Drew R. Evans, Manrico Fabretto, Pejman Hojati-Talemi, Daniel Dagnelund, Jean-Baptiste Arlin, Yves H. Geerts, Simon Desbief, Dag W. Breiby, Jens W. Andreasen,

Roberto Lazzaroni, Weimin M. Chen, Igor Zozoulenko, Mats Fahlman, Peter J. Murphy, Magnus Berggren and Xavier Crispin

Nature Materials 13, 190-194 (2014); published online 8 December 2013; corrected after print 16 April 2014.

In the version of this Article originally published, the grazing incidence wide-angle X-ray scattering (GIWAXS) pattern shown in Fig. 2c was obtained for a contaminated thin-film sample. The correct scattering pattern and caption is given here. In relation, the sentence beginning "However, the experimental scattering pattern shows pronounced off-axis scattering..." pertains to the contaminated sample and should not have been included. Furthermore, the sentence beginning "The PEDOT-Tos sample (Fig. 2c) exhibits several sharp refraction peaks..." should have begun "The PEDOT-Tos sample (Fig. 2c) exhibits several sharper peaks, and significant in-plane scattering..." In the sentence beginning "The orthorhombic unit cell suggested for..." the value of the full-width at half-maximum should have been ' $<35^{\circ}$ '. These errors do not affect the results and conclusions, and have been corrected in the online versions of the Article.

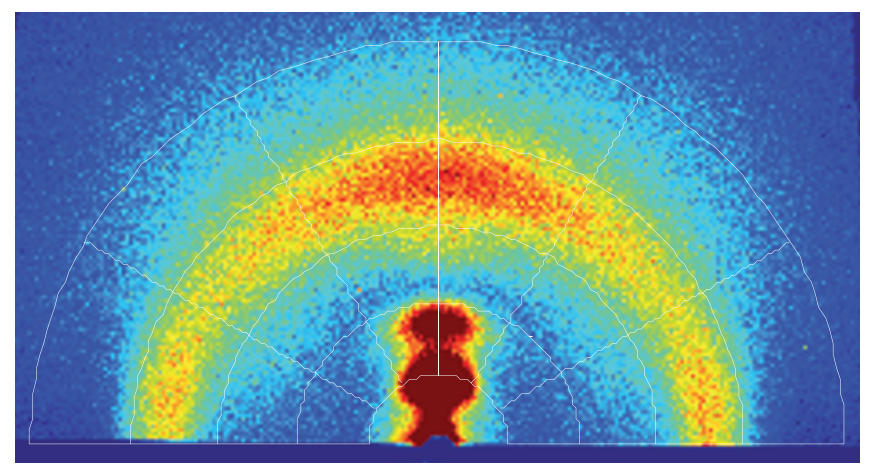

Figure $\mathbf{2}$ | c, PEDOT-Tos, being qualitatively different from the PEDOT-PSS samples, exhibits higher-order lamellae-related peaks, and also significant in-plane ordering. 